\title{
L'ambassade de Sully à Londres en 1603
}

Jean Dubu

\section{Q OpenEdition \\ Journals}

\section{Édition électronique}

URL : http://journals.openedition.org/shakespeare/501

DOI : 10.4000/shakespeare.501

ISSN : 2271-6424

Éditeur

Société Française Shakespeare

Édition imprimée

Date de publication : 1 novembre 1984

Pagination : 147-156

\section{Référence électronique}

Jean Dubu, «L'ambassade de Sully à Londres en 1603 », Actes des congrès de la Société française Shakespeare [En ligne], 5 | 1984, mis en ligne le 01 janvier 2007, consulté le 24 avril 2019. URL : http:// journals.openedition.org/shakespeare/501 ; DOI : 10.4000/shakespeare.501 
SOCIETE FRANÇAISE SHAKESPEARE

Actes du Congrès 1983

\section{MYTHE ET HISTOIRE}

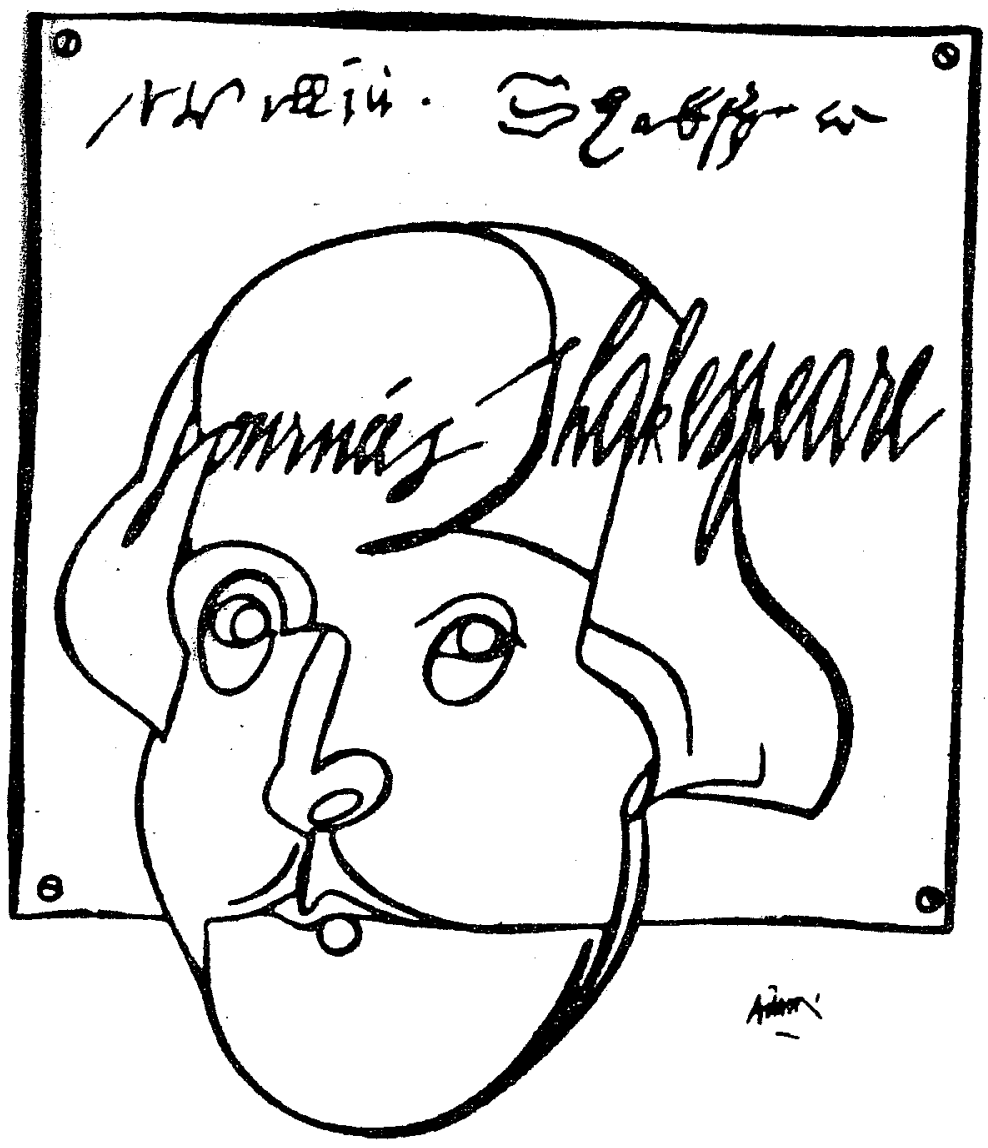

$$
\begin{array}{r}
\text { DiReCteur DE LA' PUblicatic } \\
\text { M.T. Jones-Davie }
\end{array}
$$

Publié avec le concours du Centre National de la Recherche Scientifiq

$$
\text { JEAN TOUZOT Libraire - Editeur }
$$

38, rue Saint-Sulpice 75278 PARIS CEDEX 06199 


\section{L'AMBASSADE DE SULLY A LONDRES EN 1603}

Le 24 mars 1603 à l'aube, Elizabeth Tudor, reine d'Angleterre, expire. Des relais soigneusement préparés en portent la nouvelle au roi d'Ecosse, Jacques VI Stuart. Bien que la défunte ait répugné jusqu'à la dernière extrémité à le désigner ostensiblement pour son successeur au trône d'Angleterre, il prend la route incontinent et arrive à Londres le 7 mai. Il a laissé en Ecosse la reine sa femme et leurs enfants en stipulant qu'ils ne doivent le rejoindre que sur son ordre, et que le Prince Henry, son fils aîné, futur prince de Galles, ne doit sous aucun prétexte être remis à sa mère. La raison de ces mesures qui peuvent paraître d'une exceptionnelle sévérité, sinon gravité, nous la trouvons dans une lettre de Harlay de Beaumont, ambassadeur d'Henri IV auprès d'Elizabeth, lequel, dès le 23 janvier 1603 prévenait son maître en ces termes :

... (les Anglais) étant si divisés \& si incertains en leurs affections, y ayant les uns qui désirent le Roy d'Escosse pour successeur, les autres qui le craignent \& appréhendent le gouvernement de sa femme, \& n'osent s'offrir à lui, ni se déclarer du vivant de cette Princesse... 1

A cette date, les jours d'Elizabeth âgée ne paraissent pas encore en danger immédiat ; mais à Londres, on songe sérieusement à retarder l'offre de la succession à Jacques jusqu'à la mort de ... la reine sa femme ! Hypothèse moins aventureuse qu'il pourrait le paraître à première vue, eu égard en particulier aux nombreuses grossesses de cette dernière. La prise en considération d'une telle éventualité révèle cependant un état d'esprit. Un mois plus tard, le même Beaumont revient à la charge, \& souligne :

. . je doute fort si les factions \& conjurations 
qui se découvrent \& se nourrissent continuellement en Escosse ne produisent pas bientôt quelque sinistre effet, ainsi que l'on juge par deçà que ce Prince (Jacques) ne courre une misérable fortune. Car, à ce que j'apprends de ceux qui l'affectionnent, sa femme en est le principal motif qui, pour l'espérance de gouverner seule en ce royaume sous la minorité de son fils aîné, consentira d'autant plus volontiers sa mort qu'elle y sera peutêtre poussée par quelques uns de cette Cour qui croiront devoir moins craindre le changement de leur condition sous le règne d'un enfant \& d'une femme que sous le Père, qui vraisemblablement voudra gouverner de lui-même, \& d'autant que ledit Roy est comme en sa possession, lui défère \& la croit entièrement.

Beaucoup de gens qui connaissent ses pratiques \& considèrent la conduite de sa vie \& de ses desseins doutent grandement qu'il ne mésarrive de lui par son moyen (c'est-à-dire par le moyen de la reine) \& d'ailleurs appréhendent en général son humeur que l'on dit être cruelle, brouillonne \& sanguinaire, si bien que cette crainte porte beaucoup de préjudice à l'avancement audit roi ... ${ }^{2}$

Beaumont fait également part de ses craintes au chancelier Villeroy (lequel sera sous peu mêlé à l'interdiction par le pouvoir de représenter La Royne d'Escosse de Montchrestien, tragédie peu favorable à Elizabeth); voici ce qu'il lui écrit à la fin février 1603 :

. . ce Prince (Jacques) lequel j'ai grande opinion que s'il ne se résout de le gagner de la main sur sa femme que bientôt il périsse \& reçoive le même honneur de ses prédécesseurs que les Escossois ont tués la plupart lorqu'ils ont eu des enfants, mais ce conseil est aussi violent 
comme il est peu chrestien \& ne peut lui être donné que par lui-même ... ${ }^{3}$

Le message est parfaitement reçu à Paris, d'où Henri IV répond à Beaumont, Henri bien placé pour savoir les réels dangers que court un souverain en ces temps troublés :

... Je juge avec vous digne de grande considération le péril que court le Roi d'Escosse, car s'il est vrai que sa femme ait juré sa mort, il lui sera difficile de l'éviter. S'il ne la prévient, il se montre aussi léger \& inconstant en toutes ses actions qu'il est difficile de bâtir sur ses paroles \& ses actions un solide fondement.

Il pratique à Rome, en Espagne \& partout ailleurs comme il fait avec moi, sans s'attacher à personne ouvertement ou secrètement, se mouvant \& ébranlant facilement aux premières espérances que les siens lui donnent sans en approfondir ni considérer plus avant le mérite ni la vérité.

Tellement que je prévois qu'il se laissera surprendre en toute chose... ${ }^{4}$

Ces dangers qu'il court, ce sentiment d'irrésolution qu'il donne, suscitent chez le fin politique, le Béarnais, une riposte immédiate, dès l'annonce de la mort d'Elizabeth. Rosny, futur duc de Sully, que, pour la commodité de l'exposé nous nommerons désormais Sully, Sully donc voit arriver inopinément chez lui son souverain qui lui enjoint de partir pour Londres sur-le-champ, et le charge d'une mission double : présenter ses condoléances au peuple anglais dans la personne de son nouveau souverain pour la perte de celle dont Henri ne peut oublier qu'elle a été une alliée fidèle et parfois généreuse lors de la conquête du royaume de France, et, tout en félicitant Jacques pour son accession à ce second trône, essayer de sonder les intentions politiques de cet intellectuel indécis, homme de cabinet, spéculatif. 
Le choix de Sully est habile pour cette mission délicate : sa fidélité à la Religion réformée doit créer une impression favorable auprès de ses interlocuteurs, tandis que la précédente ambassade à laquelle il a pris part à Londres lorsqu'il s'agissait de négocier un éventuel mariage entre Elizabeth et le Duc d'Alençon, frère d'Henri III, environ 1577, lui a permis de connaître le terrain. Il a aussi vu la souveraine, dans tout l'éclat de la maturité et il en garde un certain souvenir. Il est même peut-être secrètement heureux de pouvoir rendre ainsi un dernier hommage à celle que, surtout depuis l'exécution de Marie Stuart, les catholiques français ne se sont pas fait faute de vilipender, comme on peut s'en rendre compte en relisant par exemple l'Elégie écrite par Du Perron, précisément «Sur la mort de Marie Stuart».

La première des instructions fort détaillées que lui avait remises par écrit Henri IV, c'était de débuter son ambassade extraordinaire par une audience de condoléances, présentées à Jacques désormais Ier d'Angleterre en grande tenue de deuil. En langage de Versailles, SaintSimon aurait dit qu'il fallait «draper». Or, dès avant d'embarquer à Calais, voici ce que le ministre se voit contraint d'écrire à son maître :

. . . I'ay aussi appris [... ]que nul n'a apporté le deuil en Angleterre de la défuncte Reyne, ny qu'aucun Ambassadeur ne s'est présenté en cet équipage deuant le Roy qui y regne à présent ; neantmoins, considerant l'ordre \& le commandement que i'ay de vostre Majesté, \& qu'il y va bien autant de vostre dignité \& reputation, de tesmoigner encore un ressouvenir de l'amitié qu'auez portée à la defincte Reyne Elizabeth, \& des bons offices qu'en auez receus, que d'en desirer la continuation enuers ses successeurs, ie me resolus de comparoistre à la premier audience ainsi qu'il est porté par mon instruction.

Et pourtant, courtois au sens du Cortegiano de Castiglione 
\& diplomate, Sully finit par comprendre, \& cède : voici comment, dans sa lettre du 30 juin il s'en explique à Henri IV :

I'ay ci-deuant escrit à Vostre Majesté comme i'auois fait entendre à $M$. de Beaumont que i'auois charge de comparoistre en habit de dueil en ma premiere audience, \& comme il m'auoit fait responce qu'il n'estimoit pas que ce fût chose agreable ni bien receue en cette cour; me priant de lui permettre d'en escrire à ses amis, afin de ne commencer pas mon cuurage par une offense ; ce que ie trouuay fort bon, \& de fait il en escriuit au Cheualier Asquin \& autres dont il n'eust responce le Ieudy, Vendredy, ni Samedy tout le long du iour; $\mathrm{Si}$ bien que me separant le soir d'auec la noblesse qui m'accompagne (250 personnes, précisons-le), ie les priay de s'habiller tous de dueil, \& qu'il m'estoit ordonné par Vostre Majesté de comparoistre en cet Estat, à quoy chacun se resolucit, mais sur les unze heures du soir ainsy que ie me mettois au lict, Monsieur de Beaumont m'enuoya une lettre du Chevalier Asquin par laquelle il me prioit au nom de Dieu de me diuertir de ce dessein, \& d'empescher que pour chose de nulle substance, i' alterasse la bonne disposition du Roy son Maistre. I'auois desià apprins de plusieurs, mesmes du Vicomte Sauart, du Sieur de La Fontaine, des Desputez des Estats \& du sieur Stafford qui m'auoit enuoyé visiter, \& qui se monstre fort vostre seruiteur, qu'ils tenoient cette procedure comme une espece d'affront, \& un reproche public'du peu de deuoir qu'ils auoient rendu en cet endroict, $\&$ pour causer alteration parmy le peuple, luy faisant recogneistre l'obmission d'une telle ciuilité, respect \& ceremonie accoustumee entre les Princes. Sur lesquelles raisons aiant consulté auec ledict Sieur de Beaumont \& autres de vos 
seruiteurs, par leur aduis, ie me resolus de mander à la Noblesse (dont la pluspart estoient desià vestus dedueil) qu'ils eussent à changer de vestemens, ce qu'ils firent, \& chacun se para comme il pust conuertissant nostre pleur en allegresse.

Mais l'Ambassadeur ne laisse pas de remarquer à la suite :

Quoy que ce soit, tout le monde a scu le commandement que i'auois, \& que i'en ay changé la forme à l'appetit d'autruy, tellement que cela ne laissera pas de produire le mesme effect dans l'esprit des hommes, \& peutestre auec plus de vertu, encore que peu de personnes osent faire ou fassent mention de la defuncte reyne, estant sa memoire \& tous ses gestes tant recommandables enseuelis auec sa personne. Ie supplie Vostre Majesté me pardonner si en cela i'ay manqué à ses commandemens, lesquels ie n'ay pas estimé deuoir estre aussy absolus en pareille charge qu'en celle dont il luy a plu m'honorer, que les occasions \& les accidens imprimez n'y puissent changer quelque chose principalement quand elle n'est point substantielle, \& plustost pour la bienseance que pour la nécessité ou utilité qui en reuienne.

On ne s'étonnera pas que, par ce mélange de politique $\&$ de diplomatie, de franchise et de sens de la nuance, sa mission se soit trouvée facilitée. Certes, l'ambassadeur de Venise lui avait appris au cours d'une conversation que :

ce prince [Jacques] qui faisait sonner si haut \& si souuent ce grand mot de politique de l'Europe, ne s'embarrassoit de rien moins dans le fond, que toute la dissimulation dont on lui faisoit un mérite n'auoit iamais consisté qu'à donner des espérances à tout le monde et iamais d'effets à personne ; qu'il ne changeroit pas de maxime, lui 
à qui on auoit entendu dire qu'il n'y auoit que ce manège adroit, qui lui eust fait passer les dangers qu'il auoit connus, estant roy d'Escosse. . . ${ }^{7}$

Point de vue auquel Henri IV devait souscrire entièrement puisque, dans un message chiffré, il communiquait à Sully :

Le Roy d'Angleterre, comme prince d'exquise sagesse \& grandement spéculatif, \& qui mesme affecte de se faire tenir pour tel, disant qu'il estoit de la necessaire temperance \& circonspection de toute nouuelle royauté de se donner un an \& iour de terme auant que de faire aucune innouation afin de n'en exciter point qui lui puissent nuire \& semble que celles qu'il appréhende le plus doivent venir du costé de sa femme \& de son fils aîné, quoy qu'ils ayent inclinations diuerses. 8

Sully n'obtint pas moins de quatre audiences en quinze jours ; il précise dans ses Mémoires qu'il eut tout loisir d'entretenir Jacques du grand dessein politique du roi de France : constituer face à la «faction espagnole» une autre «faction» qui aurait compris, outre le roi de France, les rois d'Angleterre, d'Ecosse, d'Irlande, de Danemark et de Suède, ainsi que les villes \& communautés protestantes d'Allemagne, Suisse \& Pays-Bas, tous pays où les trois confessions calviniste, catholique \& luthérienne - par ordre alphabétique - eussent vécu en bonne intelligence ; c'était vouloir étendre à toute l'Europe du Nord le bénéfice de l'Edit de Nantes.

Comme on peut s'en douter, notre Ambassadeur n'était pas arrivé à Londres les mains vides : il y avait de l'or à répandre avec discernement auprès des diverses personnes mises à son service ; des présents aussi, appropriés à leurs destinataires, les membres de la famille royale : pour le roi. 
six très-beaux chevaux des mieux dressés fort richement enharnachés, \& le sieur Saint-An thoine pour écuyer.

ce qui n'alla pas sans difficultés pratiques, puisqu'en novembre, cinq mois après l'ambassade, trois après la signature du traité d'alliance négocié par Sully, ni les chevaux, ni l'écuyer n'étaient encore partis. Saint-Anthoine fut apprécié; il passa par la suite au service de Charles, second fils de Jacques, ainsi que l'on peut voir sur un tableau de Van Dyck actuellement dans les Collections de la Reine : Charles the First on Horseback with $M$. de Saint-Anthoine in attendance. La Catalogue de l'exposition «The Age of Charles the First» signale le personnage comme «the best master in the art of horsemanship».

Le présent destiné à la reine Anne:

.. . un miroir de Crystal de Venise dans une boëte d'or enrichie de diamants.

ceux du prince Henry :

une lance \& un heaume d'or enrichis de diamans, un escrimeur $\&$ un baladin. ${ }^{11}$

Le traité envisageait en termes très généraux «le mariage du Dauphin \& de la fille d'Henri IV avec le fils et la fille dudit Roy d'Angleterre (sic) »Vingt-deux ans plus tard, mais sans que ce fût l'effet direct de ce traité, Henriette de France devait épouser Charles Ier d'Angleterre, pour le meilleur et pour le pire... 


\author{
NOTES
}

1. Archives du Ministères des Affaires Etrangères, Paris (abrégé par la suite $A E$ ), Angleterre $24 \mathrm{f}^{\circ} 12, \mathrm{r}^{\mathrm{V}}$.

$$
\begin{aligned}
& \text { 2. AE, ibid., f } 63^{\mathrm{v}}, 64^{\mathrm{r}-\mathrm{v}} \\
& \text { 3. AE, ibid, }-\mathrm{f} 77^{\mathrm{r}} \\
& \text { 4. AE, ibid, }-\mathrm{f} 103^{\mathrm{v}}, 104^{\mathrm{r}-\mathrm{v} \text { }}
\end{aligned}
$$

5. SULLY, Mémoires des Sages et Royalles / OECONOMIES d'ESTAT / Domestiques, Politiques / Et militaires de Henry le Grand, L'exemplaire des Roys, le Prince des Vertus, / des Armes \& des Lois, \& le Père en effet de ses peuples François./

Et des Servitudes utiles, Obéissances convenables / \& administrations loyales de Maximilien de Bethune, l'un des plus confidens, / familiers et utiles soldats \& serviteurs du grand Mars des François./

Dediez à la France, à tous les bons soldats \& tous peuples François./ Grand fleuron carré avec les devises : Nusquam Marcescit Virtus

à A m stelredam Foy Esperance Charité

Chez Aletinosgraphe de Cleartimelee \& Graphexecon de Pistariste A l'en seigne des trois Vertus couronnées d'Amaranthe folio, $508+448$ p. + tables

Sur le problème délicat de la rédaction et de la publication des Mémoires de Sully, dont on vient de lire le vrai titre, nous renvoyons au livre bien documenté de B. Barbiche, Sully, Paris 1978 , notamment $\mathrm{Ch}, 1$ : "Comment connaissons-nous Sully ?»,p. 14-15.

Ce nous est un agréable devoir de remercier ici M. le Pasteur F. Delforge, conservateur de la Bibliothèque de la Société d'Histoire du Protestantisme français qui, avec beaucoup de bonne grâce, a $m$ is ces deux ouvrages à notre disposition.

t. II, p. 106 .

6. Id, ibid., t. II, p. $118-119$.

7. Id. ibid., t. II, p. 99.

8. Id.. ibid., t. 11, p. 150 .

9. Id.. ibid., t. II, p. 169.

10. Id.. ibid., t. II, p. $143 \& 145$.

11. t. II., p. 169 . 